\title{
A Universe of Binding and Computation
}

\author{
Daniel R. Licata* Robert Harper* \\ Carnegie Mellon University \\ $\{\mathrm{drl}, \mathrm{rwh}\} @ \mathrm{cs} . \mathrm{cmu}$. edu
}

\begin{abstract}
We construct a logical framework supporting datatypes that mix binding and computation, implemented as a universe in the dependently typed programming language Agda 2. We represent binding pronominally, using well-scoped de Bruijn indices, so that types can be used to reason about the scoping of variables. We equip our universe with datatype-generic implementations of weakening, substitution, exchange, contraction, and subordination-based strengthening, so that programmers need not reimplement these operations for each individual language they define. In our mixed, pronominal setting, weakening and substitution hold only under some conditions on types, but we show that these conditions can be discharged automatically in many cases. Finally, we program a variety of standard difficult test cases from the literature, such as normalization-by-evaluation for the untyped $\lambda$-calculus, demonstrating that we can express detailed invariants about variable usage in a program's type while still writing clean and clear code.
\end{abstract}

Categories and Subject Descriptors $\quad$ F.3.3 [Logics and Meanings Of Programs]: Studies of Program Constructs-Type structure

General Terms Languages, Verification

\section{Introduction}

There has been a great deal of research on programming languages for computing with binding and scope (bound variables, $\alpha$-equivalence, capture-avoiding substitution). These languages are useful for a variety of tasks, such as implementing domain-specific languages and formalizing the metatheory of programming languages. Functional programming with binding and scope involves two different notions of function: functions-as-data and functionsas-computation. Functions-as-data, used to represent abstract syntax with variable binding, have an intensional, syntactic, character, in the sense that they can be inspected in ways other than function application. For example, many algorithms that process abstract syntax recur under binders, treating variables symbolically. On the other hand, functions-as-computation, the usual functions of

\footnotetext{
* This research was sponsored in part by the National Science Foundation under grant number CCF-0702381 and by the Pradeep Sindhu Computer Science Fellowship. The views and conclusions contained in this document are those of the author and should not be interpreted as representing the official policies, either expressed or implied, of any sponsoring institution, the U.S. government or any other entity.
}

Permission to make digital or hard copies of all or part of this work for personal or classroom use is granted without fee provided that copies are not made or distributed for profit or commercial advantage and that copies bear this notice and the full citation on the first page. To copy otherwise, to republish, to post on servers or to redistribute to lists, requires prior specific permission and/or a fee.

ICFP'09, August 31-September 2, 2009, Edinburgh, Scotland, UK.

Copyright (C) 2009 ACM 978-1-60558-332-7/09/08...\$5.00 functional programming, have an extensional character-a function from $A$ to $B$ is a black box that, when given an $A$, delivers a $B$. A function-as-data determines a function-as-computation by substitution (plugging a value in for a variable), but not every functionas-computation determines a function-as-data, because the syntax appropriate for a particular problem may not allow the expression of every black box.

In previous work (Licata et al., 2008), we began to study a programming language that provides support for both functionsas-data and functions-as-computation as two different types. Our framework provides one type constructor $\Rightarrow$ for functions-as-data, used to represent variable binding, and another type constructor $\supset$ for functions-as-computation, used for functional programming. This permits representations that mix the two function spaces. As a simple example of such integration, consider a syntax for arithmetic expressions constructed out of (1) variables, (2) numeric constants, (3) let binding, and (4) arbitrary binary primitive operations, represented by functions-as-computation of type nat $\supset$ nat $\supset$ nat. In SML, we would represent this syntax with the following datatype:

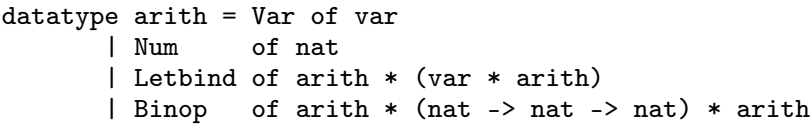

We use ML functions(-as-computation) to represent the primops. However, because SML provides no support for functions-as-data, we must represent variable binding explicitly (with a type var), and code notions such as $\alpha$-equivalence and substitution ourselves.

In contrast, our framework naturally supports mixed datatypes such as this one. We specify it by the following constructors:

$$
\begin{array}{ll}
\text { num } & : \quad \text { arith } \Leftarrow \text { nat } \\
\text { letbind }: & \text { arith } \Leftarrow \text { arith } \otimes(\text { arith } \Rightarrow \text { arith }) \\
\text { binop }: & \text { arith } \Leftarrow \text { arith } \otimes(\text { nat } \supset \text { nat } \supset \text { nat }) \otimes \text { arith }
\end{array}
$$

The symbol $\Leftarrow$ is used for datatype constructors, which have the form $D \Leftarrow A$, for a datatype name $D$ and a type $A$. We use $\Rightarrow$ (functions-as-data) to represent the body of the letbind, and $\supset$ (functions-as-computation) to represent the primops.

Our framework takes a pronominal approach to the variables introduced by functions-as-data: variables are thought of as pronouns that refer to a designated binding site, and thus are intrinsically scoped. This is in contrast to the nominal approach taken by languages such as FreshML (Pitts and Gabbay, 2000; Pottier, 2007; Shinwell et al., 2003), where variables are thought of as nounsthey are pieces of data that exist independently of any scope. The pronominal approach inherently requires some notion of context to be present in the language's type system, so that variables have something to refer to; we write $\langle\Psi\rangle A$ as the classifier of a program of type $A$ with variables $\Psi$. The practical advantage of these contextual types is that they permit programmers to express useful invariants about variable-manipulating code using the type system, such as the fact that a $\lambda$-calculus evaluator maps closed terms to closed terms. 
In a pronominal setting, the interaction of functions-as-data and functions-as-computation has interesting consequences for the structural properties of variables, such as weakening (introducing a new variable that does not occur) and substitution (plugging a value in for a variable). For example, one might expect that it would be possible to weaken a value of type $A$ to a function-asdata of type $D \Rightarrow A$. However, this is not necessarily possible when $A$ itself is a computational function type: Contextual computational functions of type $\langle\Psi\rangle A \supset B$ are essentially interpreted as functions from $\langle\Psi\rangle A$ to $\langle\Psi\rangle B$, and $\langle\Psi\rangle D \Rightarrow A$ classifies values of type $A$ in an extended context $\Psi, x: D$. Now, suppose we are given a function $f$ of type $\langle\Psi\rangle A \supset B$; we try to construct a function of type $\langle\Psi\rangle D \Rightarrow(A \supset B)$. This requires a function from $\langle\Psi, x: D\rangle A$ to $\langle\Psi, x: D\rangle B$. Since $f$ is a black box, we can only hope to achieve this by pre- and post-composing with appropriate functions. The post-composition must take $\langle\Psi\rangle B$ to $\langle\Psi, x: D\rangle B$, which would be a recursive application of weakening. However, the pre-composition has a contravariant flip: we require a strengthening function from $\langle\Psi, x: D\rangle A$ to $\langle\Psi\rangle A$ in order to call $\mathrm{f}$ - and such a strengthening function does not in general exist, because the value of type $A$ might be that very variable $x$. Similarly, substitution of terms for variables is not necessarily possible, because substitution requires weakening. Put differently, computational functions permit the expression of side conditions that inspect the context, which causes the structural properties to fail. As a concrete example, consider computational functions of type $\langle\cdot\rangle$ (arith $\supset$ arith), which are defined by case-analysis over closed arithmetic expressions, giving cases for constants and binops and let-binding-but not for variables, because there are no variables in the empty context. Weakening such a function to type arith $\Rightarrow$ (arith $\supset$ arith) enlarges its domain, asking it to handle cases that it does not cover.

What are we to do about this interaction of binding and computation? One option is to work in a less general setting, where it does not come up. For example, in nominal languages such as FreshML (Shinwell et al., 2003), the type of names is kept openended (it is considered to have infinitely many inhabitants). Thus, any computational function on syntax with binding must account for arbitrarily many names, and is therefore weakenable. However, many functions on syntax are only defined for certain classes of contexts (e.g., only closed arithmetic expressions can be evaluated to a numeral), and the nominal approach does not allow these invariants to be expressed in a program's type (though they can be reasoned about externally using a specification logic (Pottier, 2007)). Alternatively, in languages based on the LF logical framework, such as Twelf (Pfenning and Schürmann, 1999), Delphin (Poswolsky and Schürmann, 2008), and Beluga (Pientka, 2008), the structural properties always hold, because computational functions cannot be used in LF representations of logical systems.

In our framework, we take a more general approach, which requires admitting that weakening and substitution may not always be defined. Thus, we should be more careful with terminology, and say that the type $D \Rightarrow A$ classifies values of type $A$ with a free variable of type $D$. In some cases, $D \Rightarrow A$ determines a function given by substitution, but in some cases it does not. In this sense, our approach is similar to representations of binding using wellscoped de Bruijn indices (Altenkirch and Reus, 1999; Bellegarde and Hook, 1994; Bird and Paterson, 1999), which are pronominal, because variables are represented by pointers into a context, but make no commitment to weakening and substitution. However, our framework improves upon such representations by observing that weakening and substitution are in fact definable generically, not for every type $D \Rightarrow A$, but under certain conditions on the types $D$ and $A$. For example, returning to our failed attempt to weaken $A \supset B$ above, if variables of type $D$ could never appear in terms of type $A$, then the required strengthening operation would exist. As a rough rule of thumb, one can weaken with types that do not appear to the left of a computational arrow in the type being weakened, and similarly for substitution. Our framework implements the structural properties generically but conditionally, providing programmers with the structural properties "for free" in many cases. This preserves one of the key benefits of working in LF, where weakening and substitution are always defined.

In our previous work (Licata et al., 2008), we investigated the logical foundations of a pronominal approach to mixing binding and computation. In the present paper, we give an implementation of (a slight variant of) our framework, and we demonstrate the viability of our approach by programming some standard difficult test cases from the literature. For example, we implement normalization-by-evaluation (Berger and Schwichtenberg, 1991; Martin-Löf, 1975) for the untyped $\lambda$-calculus, an example considered in FreshML by Shinwell et al. (2003). Our version of this algorithm makes essential use of a datatype mixing binding and computation, and our type system verifies that evaluation maps closed terms to closed terms.

Rather than implementing a new language from scratch, we construct our type theory as a universe in Agda 2 (Norell, 2007), a dependently typed functional programming language that provides good support for programming with inductive families, in the style of Epigram (McBride and McKinna, 2004). This means that we (a) give a syntax for the types of our type theory and (b) give a function mapping the types of our language to certain Agda types; the programs of our language are then the Agda programs of those types. This implementation strategy allows us to reuse the considerable implementation effort that has gone into Agda, and to exploit generic programming within dependently typed programming (Altenkirch and McBride, 2003) to implement the structural properties; additionally, it permits programs written using our framework to interact with existing Agda code. Also, our development provides a successful example of prototyping a new language with an interesting type system using a dependently typed programming language. In our Agda implementation, we have chosen to represent variable binding using well-scoped de Bruijn indices.

In summary, we make the following technical contributions: (1) We show that our previous type theory for integrating binding and computation can be implemented as a universe in Agda. The types of the universe permit concise, "point-free" descriptions of contextual types: a type in the universe acts as a function from contexts to Agda types. (2) We implement a variety of structural properties for the universe, including weakening, substitution, exchange, contraction, and subordination-based strengthening (Virga, 1999), all using a single generic map function for datatypes that mix binding and computation. (3) We define the structural properties' preconditions computationally, so that our framework can discharge these conditions automatically in many cases. This gives the programmer free access to weakening, substitution, etc. (when they hold). (4) We program a variety of examples, and demonstrate that we can express detailed invariants about variable usage in a program's type while still writing clean and clear code.

In this paper, we consider only a simply-typed universe, for writing ML-like programs that manipulate binding in a well-scoped manner; we leave dependent types to future work. Also, the companion code for this paper (see http://www.cs. cmu . edu/ drl/) is written in "Agda minus termination checking," as many of our examples require non-termination; we discuss which parts of our code pass the termination checker below.

The remainder of this paper is organized as follows: In Section 2, we introduce our language and its semantics in Agda. In Section 3, we present examples. In Section 4, we discuss the structural properties. In Sections 5 and 6, we discuss related work and conclude. Appendix A contains a brief introduction to Agda. 


\section{Language Definition}

\subsection{Types}

The grammar for the types of our language is as follows:

$\begin{array}{llll}\text { Defined atoms } & D & :== & \ldots \\ \text { Var. Types } & C::= & \text { (a subset of } D) \\ \text { Contexts } & \Psi \quad:= & [] \mid \Psi, C) \\ \text { Types } & A \quad:== & 0^{+}\left|1^{+}\right| A \otimes B|A \oplus B| \text { list } A \mid A \supset B \\ & & \mathrm{D}^{+} D|C \#| \Psi \Rightarrow^{*} A \mid \square A \\ & & \forall_{\mathrm{c}} \psi \cdot A\left|\exists_{\mathrm{c}} \psi \cdot A\right| \forall_{\leq C} A \mid \exists_{\leq C} A\end{array}$

The language is parametrized by a class of defined atoms $D$, which are the names of datatypes. A subset of these names are variable types, which are allowed to appear in contexts. This distinguishes certain types $C$ which may be populated by variables from other types $D$ which may not. This definition of VarType permits only variables of base type, rather than the full language of higher-order rules that we considered in previous work (Licata et al., 2008). Contexts are lists of variable types, written with 'cons' on the right.

The types on the first line have their usual meaning. The type $\mathrm{D}^{+} D$ is the datatype named by $D$. Following Delphin (Poswolsky and Schürmann, 2008), we include a type $C \#$ classifying only the variables of type $C$. The type $\Psi \Rightarrow^{*} A$ classifies inhabitants of $A$ in the current context extended with $\Psi$. The type $\square A$ classifies closed inhabitants of $A$. The types $\forall_{\mathrm{c}}$ and $\exists_{\mathrm{c}}$ classify universal and existential context quantification; $\forall_{\leq C} A$ and $\exists_{\leq C} A$ provide bounded quantification over contexts containing only the type $C$.

\subsubsection{Agda implementation}

We now represent these types in Agda. Those readers who are not fluent in dependent programming can find a review of Agda syntax, well-scoped de Bruijn indices, and universes in Appendix A. We represent defined atoms, variable types and contexts as follows:

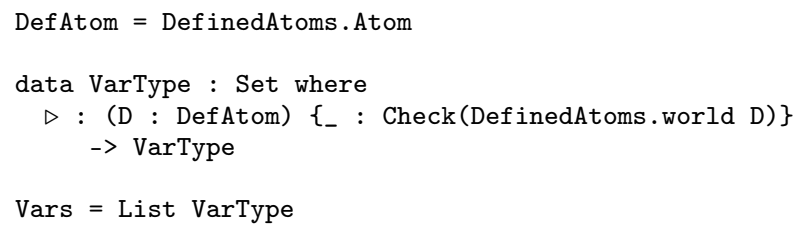

DefinedAtoms.Atom is a parameter that we will instantiate later. DefinedAtoms.world returns true when D is allowed to appear in the context; Check turns this boolean into a proposition (Check True is the unit type; Check False is the empty type; see Appendix A for an introduction). A VarType is thus a pair of an atom along with the credentials allowing it to appear in contexts.

We represent the syntax of types in Agda as follows:

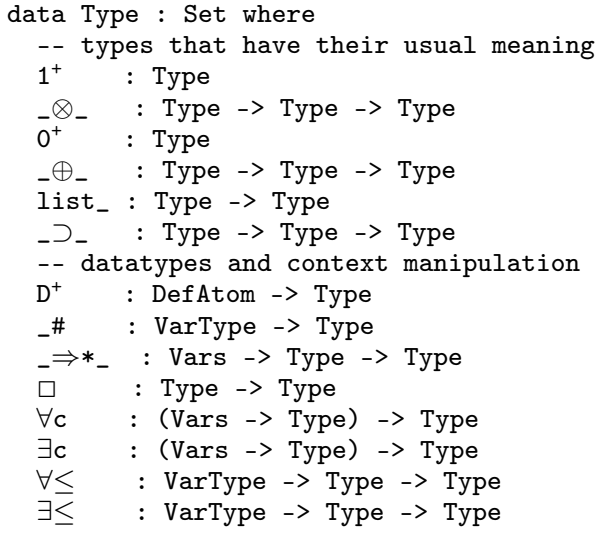

The only subtlety in this definition is that we represent the bodies of $\forall c$ and $\exists c$ by computational functions in Agda. This choice has some trade-offs: on the one hand, it means that the bodies of quantifiers can be specified by any Agda computation (e.g. by recursion over the domain). On the other hand, it makes it difficult to analyze the syntax of Types, because there is no way to inspect the body of the quantifier. Indeed, this caused problems for our implementation of the structural properties, which we solved by adding certain instances of the quantifiers $(\forall \Rightarrow$ and $\exists \Rightarrow$, discussed below), which would otherwise be derived forms, as separate Type constructors. In future work, we may pursue a more syntactic treatment of the quantifiers (which would of course be easier if we had good support for variable binding...).

A rule, which is the type of a datatype constructor, pairs the defined atom being constructed with a single premise type (no/multiple premises can be encoded using $1^{+}$and $\otimes$ ):

data Rule : Set where

$\Leftarrow_{-}$: DefAtom $->$Type $\rightarrow$ Rule

We will make use of a few derived forms:

- We write $(\forall \Rightarrow$ A) for $(\forall c \backslash \Psi->\Psi \Rightarrow * A)$, and similarly for $\exists \Rightarrow$ (note that $\backslash \mathrm{x} \rightarrow$ e introduces an anonymous function). This type quantifies over a context $\Psi$ and immediately binds it around A. Similarly, we write $[\Psi] *$ A for $\square(\Psi \Rightarrow *$ A)

- We write $(\mathrm{C} \Rightarrow \Psi$ ) for $\Rightarrow *$ with a single premise.

- We write $\left(\mathrm{C}^{+}\right)$for $\left(\mathrm{D}^{+} \mathrm{C}\right)$ when $\mathrm{C}$ is a variable type.

- We write bool for $1^{+} \oplus 1^{+}$and $\mathrm{A}$ option for $\mathrm{A} \oplus 1^{+}$.

\subsection{Semantics}

A universe is specified by a inductive datatype of codes for types, along with a function mapping each code to a Set. In this case, the Types above are the codes, and the semantics is specified in Figure 1 by a function $\langle\Psi\rangle$ A, mapping a context and a Type to an Agda Set. The first six cases interpret the basic types of the simply-typed $\lambda$-calculus as their Agda counterparts, pushing the context inside to the recursive calls.

The next two cases interpret datatypes. We define an auxiliary datatype called Data which represents all of the data types defined in the universe. Data is indexed by a context and a defined atom, with the idea that the Agda set Data $\Psi$ D represents the values of datatype $\mathrm{D}$ in context $\Psi$. For example, the values of Data $\Psi$ arith will represent the arithmetic expressions defined by the signature given in the introduction. There are two ways to construct a datatype: (1) apply a datatype constructor to an argument and (2) choose a variable from $\Psi$. Constants are declared in a signature, represented with a predicate on rules $\operatorname{In} \Sigma:$ Rule $\rightarrow$ Set, where $\operatorname{In} \Sigma \mathrm{R}$ is inhabited when the rule $\mathrm{R}$ is in the signature. The first constructor, written as infix $\cdot$, pairs a constant with the interpretation of the constant's premise. The second constructor, $\triangleright$, injects a variable from $\Psi$ into Data. ${ }^{1}$ See the appendix for the definition of the type $\in$, which represents well-scoped de Bruijn indices (Altenkirch and Reus, 1999; Bellegarde and Hook, 1994; Bird and Paterson, 1999). A DefAtom D is in the context if there exist credentials $c$ for which the VarType formed by ( $\triangleright \mathrm{D}\{\mathrm{c}\}$ ) is in the list $\Psi$.

Finally, we provide a collection of types that deal with the context: $\Psi \Rightarrow *$ A extends the context (we write + for append); $\square$ A clears the context. The quantifiers $\forall c$ and $\exists c$ are interpreted as the corresponding Agda dependent function and pair types. Finally,

\footnotetext{
${ }^{1}$ Agda allows overloading of datatype constructors between different types, and we tend to use $\triangleright$ for injections from one type to another, as with VarType above.
} 


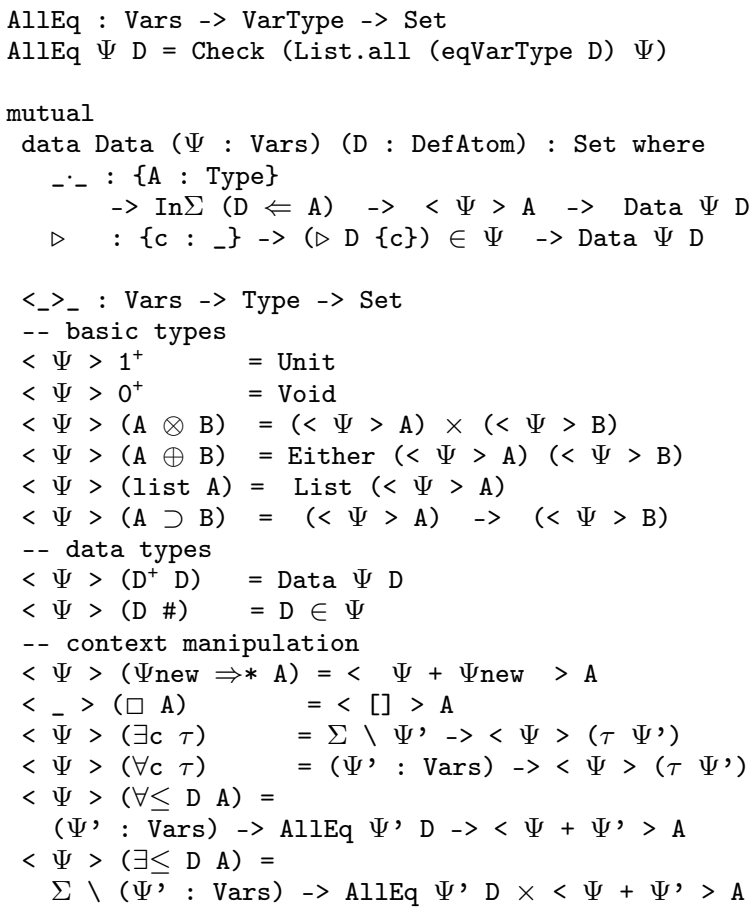

Figure 1. Semantics

the types $\forall \leq \mathrm{D}$ A and $\exists \leq \mathrm{D}$ A quantify over contexts $\Psi$, for which AllEq $\Psi$, D holds. The type AllEq says that every variable type in $\Psi$ is equal to the given type $\mathrm{D}$ (List.all is true when its argument is true on all elements of the list; eqVarType is a boolean-valued equality function for variable types). (We could internalize $\mathrm{AllEq} \Psi, \mathrm{D}$ as a type alleq $\mathrm{D}$-given meaning by $\langle\Psi\rangle$ (alleq D) $=$ AllEq $\Psi$ D-in which case the bounded quantifier could expressed as a derived form, but we have not needed alleq $\mathrm{D}$ in a positive position in the examples we have coded so far.)

An Agda datatype is strictly positive if it does not appear to the left of any Agda function types $(->)$ in its own definition; this positivity condition ensures that the user does not define general recursive types (e.g. $\mu D . D \rightarrow D$ ), which can be used to inhabit any type and to write non-terminating code. The above type Data does not pass the positivity checker: it is defined mutually with $<_{-}>_{-}$, and $<_{-}>_{-}$occurs to the left of an Agda function type in the meaning of $\supset$. In this paper, we wish to program with general recursive types, so we will ignore this failure of positivity checking. An interesting direction for future work would be to consider a total variant of our framework, which admits only strictly positive types. This would require a more refined explanation of the construction of the defined atoms in the universe, e.g. using containers (Abbott et al., 2005), because the positivity of a defined atom D depends on the rules for $\mathrm{D}$ in the signature $\operatorname{In} \Sigma$.

We also define versions of $\square$ and $\forall \Rightarrow$ that construct Agda Sets, so that we do not need to write $<[]>\square$ A and so on as the Agda type of a term. (We intentionally use a very similar notation for these; to a first approximation, one can read our examples without keeping this distinction in mind.)

$$
\begin{aligned}
& \square: \text { Type }->\text { Set } \\
& \square \mathrm{A}=\langle[]>\mathrm{A} \\
& \forall \Rightarrow-: \text { Type }->\text { Set } \\
& \forall \Rightarrow-\mathrm{A}=(\Psi \text { : Vars })->\langle\Psi>\mathrm{A}
\end{aligned}
$$

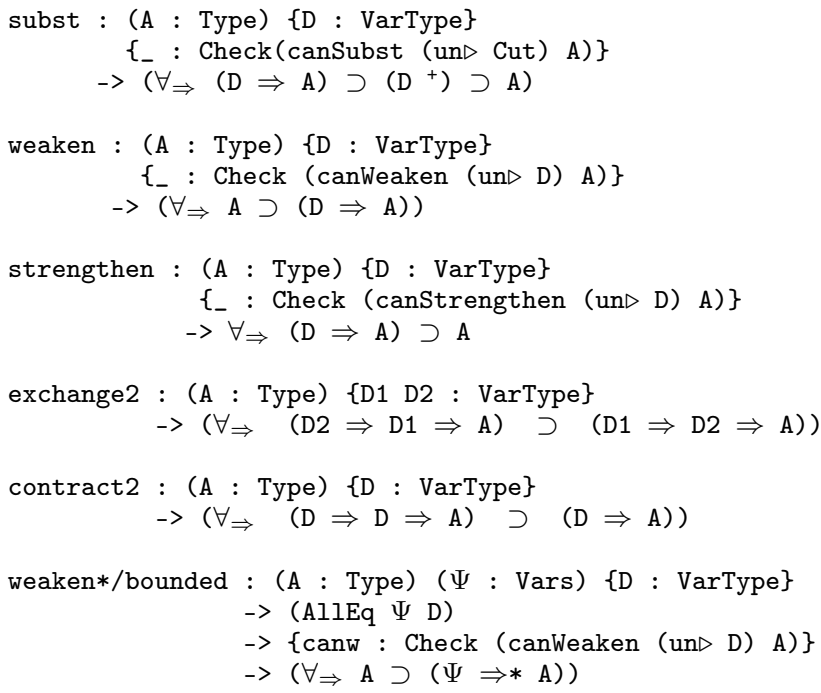

Figure 2. Type signatures of structural properties

\subsection{Structural Properties}

In Figure 2, we present the type signatures for the structural properties; this is the interface that users of our framework see.

For example, the type of substitution should be read as follows: for any $A$ and $D$, if the conditions for substitution hold, then there is a function of type $\left(\forall \Rightarrow(D \Rightarrow A) \supset\left(D^{+}\right) \supset A\right)$ (for any context, given a term of type $A$ with a free variable, and something of type $\mathrm{D}^{+}$to plug in, there is a term of type A without the free variable). Weakening coerces a term of type A to a term with an extra free variable; strengthening does the reverse; exchange swaps two variables; contraction substitutes a variable for a variable. We also include an $n$-ary version of weakening for use with the bounded quantifier: if $\mathrm{A}$ can be weakened with $\mathrm{D}$, then $\mathrm{A}$ can be weakened with a whole context comprised entirely of occurrences of D.

We discuss the meaning of the conditions (canSubst, etc.) below; in all of our examples, they will be discharged automatically by our implementation.

\section{Examples}

In this section, we illustrate programming in our framework, adapting a number of examples that have been considered in the literature (Pientka, 2008; Poswolsky and Schürmann, 2008; Shinwell et al., 2003). Throughout this section, we compare the examples coded in our framework with how they are/might be represented in Twelf, Delphin, Beluga, and FreshML. We endeavor to keep these comparisons objective, focusing on what invariants of the code are expressed, and what auxiliary functions the programmer needs to define. Aside from Twelf, we are not expert users of these other systems, and we welcome feedback from those who are. Several additional examples are available in the companion Agda code, including a translation from $\lambda$-terms to combinators, a type checker for simply-typed $\lambda$-calculus terms, an evaluator for $\lambda$-calculus with mutable references (using variables to represent locations), and an alternate version of normalization-by-evaluation, which has simpler types at the expense of slightly more-complicated code.

To use our framework, we give a type Def Atom representing the necessary datatypes names, along with a datatype

data $\operatorname{In} \Sigma$ : Rule $\rightarrow$ Set where

defining the datatype constructors. 
We use the following naming convention: Defined atoms are given names that end in A; e.g., for the signature for arithmetic expressions given in the introduction, we will define natA and arithA. For types of variables, we define $<$ atom $>C$ to be $<$ atom $>A$ injected into VarType:

arithC $=\triangleright$ arithA

We define <atom $>$ to be the Type constructed by $\mathrm{D}^{+}<$atom $>$A; e.g.:

nat $=\mathrm{D}^{+}$nat $\mathrm{A}$

arith $=D^{+}$arithA

\subsection{Evaluating Arithmetic Expressions}

We define a signature for the arithmetic example mentioned above:

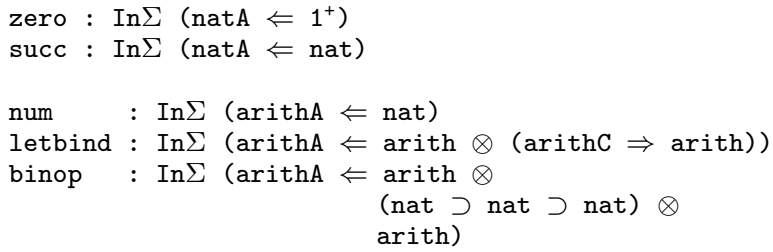

Natural numbers are specified by zero and successor. Arithmetic expressions are given as a mixed datatype, with $\Rightarrow$ used to represent the body of the letbind and $\supset$ used to represent primops.

Next, we define an evaluation function that reduces an expression to a number:

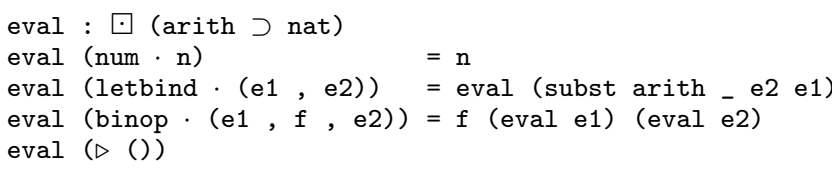

Evaluation maps closed arithmetic expressions to natural numbers (the type expression $\square$ (arith $\supset$ nat) reduces to the Agda function type Data [] arithA $\rightarrow$ Data [] natA). Constants evaluate to themselves; binops are evaluated by applying their code to the values of the arguments; let-binding is evaluated by substituting the expression e 1 into the letbind's body e $2^{2}$ and then evaluating the result. A simple variation would be to evaluate $\mathrm{e} 1 \mathrm{first}$ and then substitute its value into e2. The final clause covers the case for variables with a refutation pattern: there are no variables in the empty context.

Comparison. This example provides a nice illustration of the benefits of our approach: Substitution is provided "for free" by the framework, which infers that it is permissible to substitute for arithC variables in arith. The type system enforces the invariant that evaluation produces a closed natural number.

It is not possible to define the type arith in Twelf/Delphin/Beluga, as LF representations cannot use computational functions. One could program this example in FreshML, but it would be necessary to implement substitution directly for arith, as FreshML does not provide a generic substitution operation.

Agda checks that eval's pattern matching is exhaustive. However, Agda is not able to verify the termination of this function, as it recurs on a substitution-instance of one of the inputs. Setting aside the computational functions in binop, it would be possible to get the call-by-value version of this code to pass Twelf's termination

\footnotetext{
${ }^{2}$ The arith argument to subst is the type $\mathrm{A}$ in the $\mathrm{D} \Rightarrow \mathrm{A}$ argument to substitution; Agda's type reconstruction procedure requires this annotation. The underscore is the context argument instantiating the $\forall \Rightarrow$ in the type of subst; this could be eliminated by adding an implicit context quantifier (whose meaning is $\{\Psi$ : Vars $\}->\ldots$ ) to the universe. The credentials for performing substitution are marked as an implicit argument, so there is no evidence of it visible in the call to subst.
}

checker, which recognizes certain substitution instances as smaller. We have not yet investigated how to explain this induction principle to Agda.

\subsection{Closure-based Evaluator}

Next, we implement a closure-based evaluator for the untyped $\lambda$ calculus. $\lambda$-terms and closures are represented by types exp and clos as follows:

$$
\begin{aligned}
& \operatorname{lam}: \operatorname{In} \Sigma(\operatorname{expA} \Leftarrow(\operatorname{expC} \Rightarrow \exp )) \\
& \text { app }: \operatorname{In} \Sigma(\operatorname{expA} \Leftarrow \exp \otimes \exp ) \\
& \text { closure }: \operatorname{In} \Sigma(\operatorname{clos} A \Leftarrow(\exists \Rightarrow \quad(\operatorname{expC} \Rightarrow \exp ) \otimes \\
& \quad(\operatorname{expC} \# \supset \text { clos })))
\end{aligned}
$$

Expressions are defined by the usual signature, as in LF. The type of closures, clos, is a recursive type with one constructor closure. The premise of closure should be read as follows: a closure is constructed from a triple $(\Psi, \mathrm{e}, \sigma)$, where (1) $\Psi$ is an existentially quantified context; (2) e is an expression in $\Psi$ with an extra free variable, which represents the body of a $\lambda$-abstraction; and (3) $\sigma$ is a substitution of of closed closures for all the variables in $\Psi$. We represent a substitution as a function that maps each expression variable in the context (classified by the type expC \#) to a closure. The type of the premise provides a succinct description of all of this: $\exists \Rightarrow$ introduces the variables in the existentially quantified context into scope without explicitly naming the context; $\Rightarrow$ extends the context with an additional variable; $(\operatorname{expC} \#)$ ranges over all of the variables in scope. For comparison, in $\Psi$ this type reduces to the Agda type

$$
\begin{aligned}
\Sigma \backslash(\Psi,: \text { Vars }) \rightarrow & (\text { Data }(\Psi+\Psi,, \operatorname{expC}) \operatorname{expA}) \times \\
& \left(\operatorname{expC} \in\left(\Psi+\Psi^{\prime}\right) \rightarrow \text { Data [] closA }\right)
\end{aligned}
$$

(where we write ,, for cons on the right).

In this example, unlike the above evaluator for closed arithmetic expressions, we recur over open expressions, so eval is quantified over an unknown context $\Psi$ using $\forall \Rightarrow$. Evaluation takes two further arguments: (1) an expression with free variables in $\Psi$, and (2) an environment, represented by a function that yields a closed closure for each expression variable in $\Psi$; eval returns a closed closure.

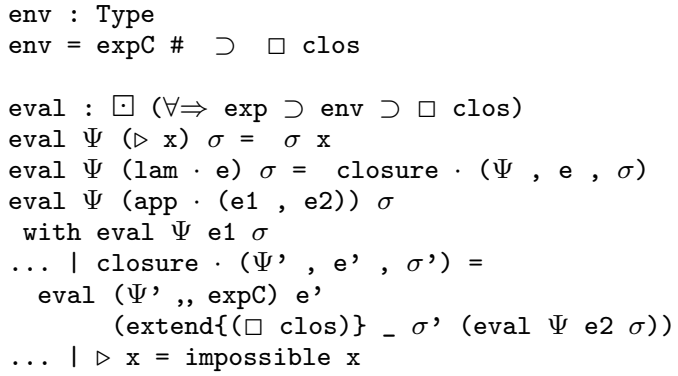

A variable is evaluated by applying the substitution. A lam evaluates to the obvious closure. To evaluate an application, we first evaluate the function position. To a first approximation, the reader may think of Agda's with syntax as a case statement in the body of the clause, with each branch marked by ... I. Case-analyzing the evaluation of e 1 gives two cases: (1) the value is constructed by the constructor closure; (2) the value is a variable.

In the first case, we evaluate the body of the closure in an extended environment. The call to the function extend extends the environment $\sigma$ 'so that the last variable is mapped to the value of e2. The definition of extend is as follows:

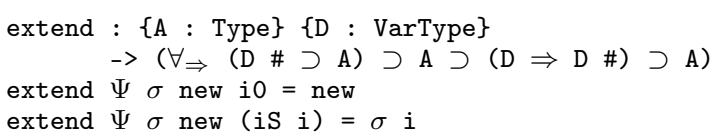


At the call site of extend, we must explicitly supply the type A (in this case $\square$ clos) to help out type reconstruction. The underscore stands for the instantiation of the $\forall \Rightarrow$, which is marked as an explicit argument, but can in this case be inferred.

The second case is contradicted using the function impossible, which refutes the existence of a variable at a non-VarType-which clos is, because we never wish to have clos variables.

The context argument $\Psi$ to eval does not play an interesting role in the code, but Agda's type reconstruction requires us to supply it explicitly at each recursive call. In future work, we may consider whether this argument can be inferred. Agda is unable to verify the termination of this evaluator for the untyped $\lambda$-calculus, as one would hope.

When writing this code, one mistake a programmer might make is to evaluate the body of the closure in $\sigma$ instead of $\sigma^{\prime}$, which would give dynamic scope. If we make this mistake, Agda highlights the occurrence of $\sigma$ and helpfully reports the type error that $\Psi, \quad !=\Psi$, indicating that the context of the expression does not match the context of the substitution.

Comparison. In Twelf, one cannot represent substitutions $\sigma$ using computational functions, because these are not available for use in LF encodings. However, because the domain of the substitution is finite, a first-order representation of substitutions could be used. Additionally, Twelf does not provide the $\square$ and $\exists \Rightarrow$ connectives that we use here to describe the contexts of closures. While it should be possible for the programmer to express the necessary context invariants using explicit contexts (Crary, 2008), this is a fairly heavy encoding technique. Because of these two limitations, the resulting Twelf code would be more complicated than the above. One would hope for better Delphin and Beluga implementations than a port of the Twelf code, but Delphin lacks existential context quantification and $\square$, and Beluga lacks the parameter type $\exp \#$, so our definition of clos cannot be straightforwardly ported to either of these languages. ${ }^{3}$ One could implement this example in FreshML (Shinwell et al., 2003), but the type system would not enforce the invariant that closures are in fact closed. To our knowledge, a proof of this property for this example has not been attempted in Pure FreshML (Pottier, 2007), though we know of no reason why it would not be possible.

\subsection{Variable Manipulation}

Next, we consider a suite of simple variable manipulations.

\subsubsection{Size}

First, we compute the size of a $\lambda$-term. Addition is defined as usual, with a contradictory variable case because no natA variables are allowed.

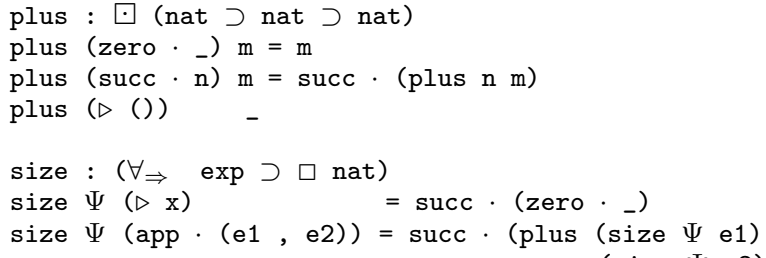

Agda successfully termination-checks these functions.

\footnotetext{
${ }^{3}$ Beluga provides a built-in type of substitutions, written $\left[\Psi^{\prime}\right] \Psi$, so one might hope to represent closures as $\exists \psi \cdot([\psi, x$ : exp]exp $) \times[.] \psi$; however, the second component of this pair associates an expression with each expression variable in $\psi$, whereas, in this example, we need to associate a closure with each expression variable in $\psi$.
}

The type of size expresses that it returns a closed natural number. For comparison, we implement a second version that does not make this invariant explicit:

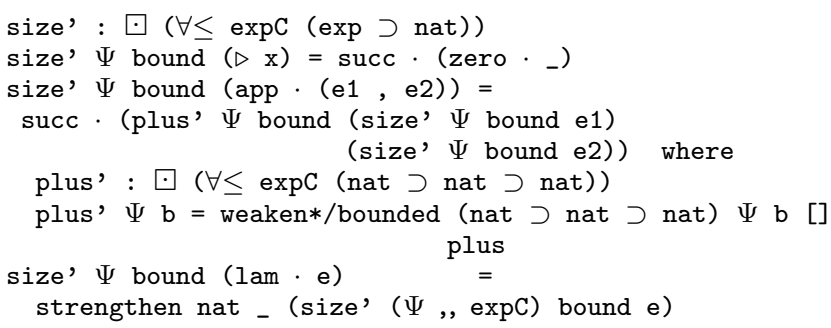

Without the $\square$, size must return a number in context $\Psi$ : in the application case, we must weaken plus into $\Psi$, and in the lam case we must strengthen the extra expC variable out of the recursive call. Strengthening expression variables from natural numbers is permitted by our implementation of the structural properties because natural numbers cannot mention expressions; we use a subordinationlike analysis to determine this (Virga, 1999). To ensure that these weakenings and strengthenings are permitted, we type size' with a bounded quantifier over exp.

Comparison. The first version is similar to what one writes in FreshML, except in that setting there is no need to pass around a context $\Psi$. In the second version, the strengthening of the recursive result in the lam case is analogous to the need, in FreshML 2000 (Pitts and Gabbay, 2000), to observe that nat is pure (always has empty support); FreshML (Shinwell et al., 2003) does not require this.

In Beluga, one can express either the first or second versions. In Twelf and Delphin, one can only express the second variation, as these languages do not provide $\square$. However, the Twelf/Delphin/Beluga syntax for weakening and strengthening is terser than what we have been able to construct in Agda: weakening is handled by world subsumption and is not marked in the proof term; strengthening is marked by pattern-matching the result of the recursive call and marking those variables that do occur, which in this case does not include the expression variable. For example, the lam case of size in Twelf looks like this:

- $: \operatorname{size}(\operatorname{lam}([\mathrm{x}] \mathrm{E} \mathrm{x}))(\operatorname{succ} N)$

$<-(\{x: \exp \} \operatorname{size}(E x) N)$.

Twelf's coverage checker verifies that expression variables can be strengthened out of natural numbers when checking this case. We would like to explore a similarly terse syntax for weakening/strengthening in future work.

\subsubsection{Counting occurrences of a variable}

A simple variation is to count the number of occurrences of a distinguished free variable. The input to this function has type (expC $\Rightarrow \exp$ ), and we count the occurrences of the bound variable:

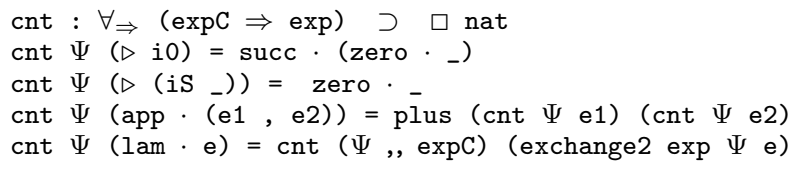

In the first two cases, we pattern-match on the variable: when it is the last variable, the last variable occurs once; when it is not, it occurs zero times. The lam case recurs on the exchange of e, so that the last variable remains the one we are looking for. Agda fails to termination-check this example because it recurs on the result of exchange. Because this use of exchange is a common recursion pattern for (exp $\rightarrow$ exp) in Twelf, we plan to consider a derived induction principle that covers this case in future work. 
Comparison. Pattern-matching on variables is represented using higher-order metavariables in Twelf/Delphin/Beluga and using equality tests on names in FreshML. The exchange needed in the lam case is written as a substitution in the Twelf/Delphin/Beluga version of this clause. In Twelf one would write:

- : $\operatorname{cnt}([\mathrm{x}] \operatorname{lam}([\mathrm{y}] \mathrm{E} \mathrm{x} y)) N$

$<-(\{y: \exp \} \operatorname{cnt}([\mathrm{x}] \mathrm{E} \mathrm{x} y) N)$.

In the input to this clause, the metavariable E, which stands for the body of the function, refers to the last variable in the context (the lam-bound variable) as $y$ and the second-last variable (the variable being counted) as $x$. In the recursive call, $y$ is exchanged past the binding of $\mathrm{x}$, so the instantiation $\mathrm{E} \mathrm{x}$ y swaps "last" and "secondlast".

\subsubsection{Computing free variables}

Next, we consider a function computing the free variables of an expression, of type $(\forall \Rightarrow \exp \supset$ list $(\operatorname{expC} \#))$ - in any context, this function accepts an expression in that context and produces a list of variables in that context. This typing ensures that we do not accidentally return a bound variable.
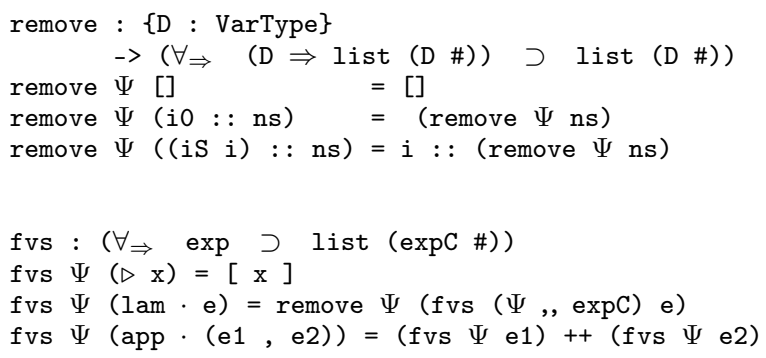

In the lam case, we use the helper function remove to remove the lam-bound variable from the recursive result. The function remove takes a list of variables, itself with a distinguished free variable, and produces a list of variables without the distinguished variable. If the programmer were to make a mistake in the second clause by accidentally including io in the result, he would get a type error. Agda successfully termination-checks this example.

Comparison. For comparison with FreshML (Shinwell et al., 2003), the type given to remove here is analogous to their Figure 6:

remove : (<name> (name list)) $->$ name list

where $<\mathrm{a}>\tau$ is a nominal abstractor. The authors comment that they prefer the version of remove in their Figure 5:

remove : name $->$ (name list) $\rightarrow$ name list

where the name to removed is specified by the first argument, rather than using a binder.

Using dependent types, we can type this second version of remove as follows:

remove : $(\Psi: \operatorname{Vars})(i: \exp \in \Psi)$

$$
\rightarrow \text { List }(\exp \in \Psi) \rightarrow \text { List }(\exp \in(\Psi-i))
$$

where $\Psi$ - i removes the indicated element element from the list. This type is of course expressible in Agda, but we have not yet integrated dependent types into our universe.

\subsection{4 $\eta$-Contraction}

In Twelf/Delphin/Beluga, one can recognize $\eta$-redices by writing a meta-variable that is not applied to all enclosing locally bound variables. E.g. in Twelf one would write

- : contract (lam $[\mathrm{x}]$ app F x) F.
The metavariable $\mathrm{F}$ : exp is bound outside the scope of $\mathrm{x}$, and thus stands only for terms that do not mention $\mathrm{x}$. (To allow it to mention $\mathrm{x}$, we would bind $\mathrm{F}: \exp \rightarrow \exp$ and write $(\mathrm{F} x)$ in place of $\mathrm{F}$.)

Unfortunately, Agda does not provide this sort of pattern matching for our encoding - pattern variables are always in the scope of all enclosing local binders-so we must explicitly call a strengthening function that checks whether the variable occurs:

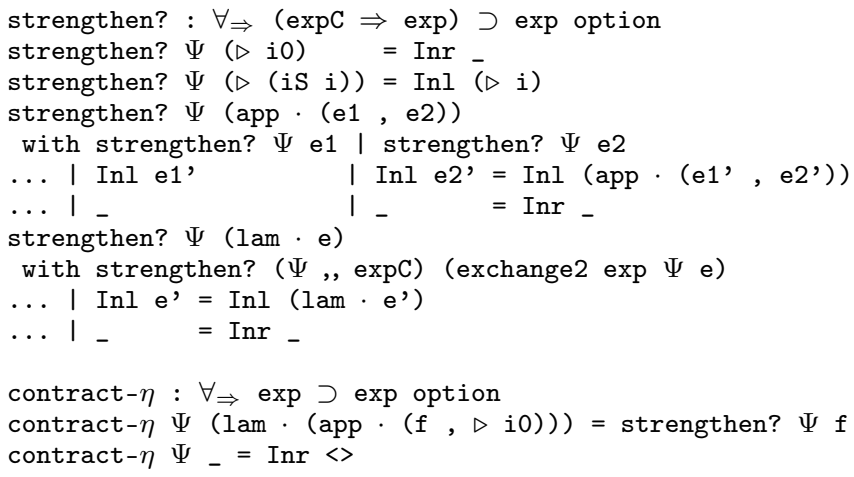

We conjecture that strengthen? could be implemented datatypegenerically for all purely positive types (no $\supset$ or $\forall \mathrm{c}$ or $\forall \leq$ ) —it is not possible to decide whether a variable occurs in the values of these computational types (cf. FreshML, where it is not possible to decide whether a name is in the support of a function). This strengthening function is not an instance of the generic map that we define below, as it changes the type of the term (exp to exp option); in future work, we plan to consider a more general traversal that admits this operation.

\subsection{Normalization by Evaluation}

In Figure 3, we present a serious example mixing binding and computation, $\beta$-normalization-by-evaluation for the untyped $\lambda$ calculus. NBE works by giving the syntax a semantics in terms of computational functions (evaluation) and then reading back a normal form (reification). The NBE algorithm is similar to a Kripke logical relations argument, where one defines a type- and context-indexed family of relations $\llbracket A \rrbracket$ in $\Psi$. The key clause of this definition is:

$$
(\llbracket A \text { arrow } B \rrbracket \text { in } \Psi)=\forall \Psi^{\prime} .\left(\llbracket A \rrbracket \text { in } \Psi, \Psi^{\prime}\right) \supset\left(\llbracket B \rrbracket \text { in } \Psi, \Psi^{\prime}\right)
$$

That is, the meaning of $A$ arrow $B$ in $\Psi$ is a function that, for any future extension of the context, maps the meaning of $A$ in that extension to the meaning of $B$ in that extension. In our type theory, we represent (a simply-typed version of) this logical relation as a datatype sem. The datatype constructor corresponding to the above clause would have the following type:

sem $\Leftarrow(\forall \Rightarrow$ sem $\supset$ sem $)$

However, for the argument to go through, we must ensure that the context extension $\Psi$ ' consists only of variables of a specific type neu, so we use a bounded context quantifier below.

We represent the semantics by the datatypes neu and sem in Figure 3. The type neu (neutral terms) consists of variables or neutral terms applied to semantic arguments (napp); these are the standard neutral proofs in natural deduction. A sem (semantic term) is either a neutral term or a semantic function. A semantic function of type $(\forall \leq$ neuC (sem $\supset$ sem)) is a computational function that works in any extension of the context consisting entirely of neu variables.

We define reification first, via two mutually recursive functions, reifyn (for neutral terms) and reify (for semantic terms). It is typical in logical relations arguments to use two independent contexts, one for the syntax and one for the semantics. Thus, we 
tier, 2007), but we would like to enforce these invariants within a type system, not using an external specification logic. Relative to a direct implementation in Agda, our framework provides the weakening function needed in the final case of eval for free.

\section{Structural Properties}

The structural properties are implemented by instantiating a generic traversal for $\langle\Psi\rangle$ A. The generic traversal has the following type:

$$
\begin{aligned}
\operatorname{map} & : \text { (A : Type) }\left\{\Psi \Psi^{\prime}: \text { Vars }\right\} \\
& \rightarrow\left(\text { (Co A } \Psi \Psi^{\prime}\right) \rightarrow\langle\langle\Psi\rangle \text { A } \rightarrow\langle\Psi,>\text { A }
\end{aligned}
$$

This should be read as follows: for every A $\Psi \Psi^{\prime}$, under the condition Co A $\Psi \Psi^{\prime}$, there is a map from terms of type A in $\Psi$ to terms of type $\mathrm{A}$ in $\Psi$,

Co : Type $\rightarrow$ Vars $\rightarrow$ Vars $\rightarrow$ Set is a variable relation, a type-indexed family of relations between two contexts. Co is in fact a (module-level) parameter to the generic map; it must provide (1) a variable or term in $\Psi$ ' for each variable in $\Psi$ that the traversal runs into; and (2) enough information to keep the traversal going inductively. We will instantiate Co with a specific relation for each traversal; e.g., for weakening with a variable of type $D$, Co will relate $\Psi$ to $(\Psi$, , D) under appropriate conditions on D and A.

For expository purposes, we present a slightly simplified version of the traversal first; the generalization is described with weakening below.

\subsection{Compatibility}

We ensure that Co provides the two pieces of information mentioned above using the notion of compatibility. Suppose that Co and Contra are variable relations. We say that Co and Contra are compatible iff there is a term

$$
\text { compat : } \begin{aligned}
\left(\{\mathrm{A}: \text { Type }\}\left\{\Psi \Psi^{\prime} \text { : Vars }\right\}\right. \\
\left.->\text { Co A } \Psi \Psi^{->} \text {Compat A } \Psi \Psi^{\prime}\right)
\end{aligned}
$$

where Compat is defined as follows:

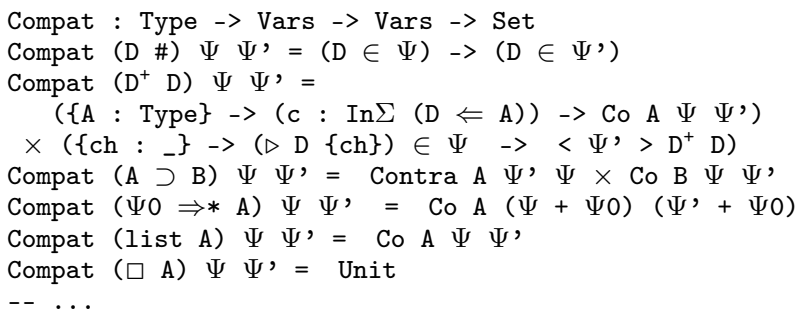

Compat imposes certain conditions on Co and Contra. For example, for variable types $\mathrm{D}$ \#, it says that Co (D \#) $\Psi \Psi$ ' induces a map from variables of type $\mathrm{D}$ in $\Psi$ to variables in $\Psi^{\prime}$. For defined atoms $\mathrm{D}^{+} \mathrm{D}$, Compat says that Co $\left(\mathrm{D}^{+} \mathrm{D}\right) \Psi \Psi$, induces a map from variables in $\Psi$ to terms in $\Psi^{\prime}$, and that Co A $\Psi \Psi^{\prime}$ ' holds for every premise A of every constant inhabiting D. In all other cases, Compat provides enough information to keep the induction going in map below. This amounts to insisting that Co (or Contra) holds on the subexpressions of a type in all appropriate contexts. For example, the condition for $\Psi 0 \Rightarrow * A$ is that Co holds for $\mathrm{A}$ in the contexts extended with $\Psi 0$.

In the usual monadic traversals of syntax (Altenkirch and Reus, 1999), Co $-\Psi \Psi^{\prime}$ is taken to be (D : VarType) $\rightarrow \mathrm{D} \in \Psi$ $\rightarrow<\Psi,>$ D - i.e. a realization of every variable in $\Psi$ as a term in $\Psi^{\prime}$. In our setting, this does not suffice to define a traversal, because (1) it does not provide for the contravariant flip necessary to process the domains of computational functions and (2) it does not allow us to express a conditional traversal, where conditions on the

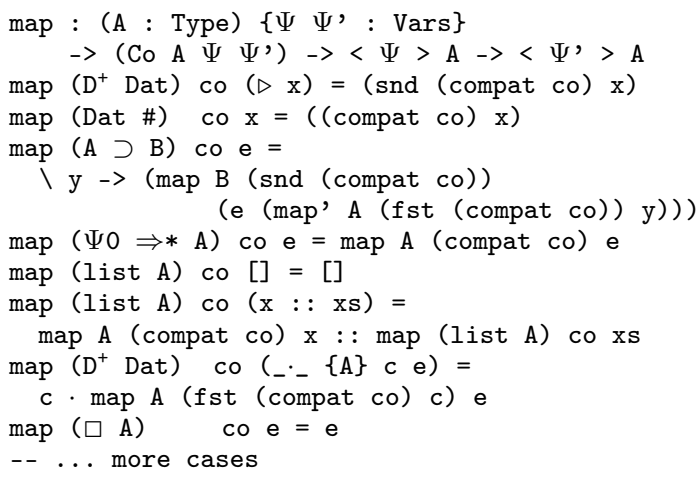

Figure 4. Map (excerpt)

types ensure that the traversal will only find certain variables, and thus that only those variables need realizations. Compatibility ensures that Co provides enough information for Contra to process the contravariant positions to the left of a computational arrow. Additionally, it permits conditional traversals: below, we will instantiate Co so that it is uninhabited for certain A.

\subsection{Map}

Suppose that Co and Contra are compatible, and assume a function map' : (A : Type) $\{\Psi \Psi$, : Vars $\}$ -> (Contra A $\left.\left.\Psi \Psi^{\prime}\right)-\right\rangle\langle\Psi\rangle$ A -$\rangle\langle\Psi$, $\rangle$ A

that is the equivalent of map for the Contravariant positions.

Then we implement map in Figure 4. In the first and second cases, the compatibility of Co induces the map on variables that we need. In the third case, we pre-compose the function with map' and post-compose with map. In all other cases, map simply commutes with constructors, or stops early if it hits a boxed term.

\subsection{Exchange/Contraction}

Exchange and contraction are implemented by one instantiation of map. In this case, we take

Co A $\Psi \Psi^{\prime}=$ Contra A $\Psi \Psi^{\prime}=\left(\Psi \subseteq \Psi^{\prime} \times \Psi^{\prime} \subseteq \Psi\right)$

where $\subseteq$ means every variable in one context is in the other. It is simple to show that these relations are compatible, because Co (a) provides the required action on variables directly and (b) ignores its type argument, so the compatibility cases for the type constructors are easy. Exchange is defined by instantiating the generic map with Co, where map' is taken be map itself, which works because Co $=$ Contra.

\subsection{Strengthening}

Next, we define a traversal that strengthens away variables that, based on type information, cannot possibly occur. The invariant for strengthening is the following: ${ }^{4}$

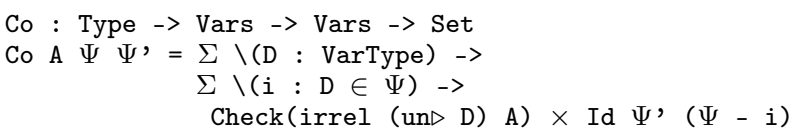

Here $i$, a pointer into the initial context $\Psi$ is the variable to be strengthened away; the propositional equality constraint represented by the Identity says that the final context $\Psi$ ' is the initial context with i removed. The type Check (irrel (unD D) A)

\footnotetext{
${ }^{4}$ For concision, we suppress some details arising from the implementation of irrel, which takes a visited list as an extra argument; see the companion code for details.
} 
computes to Unit when strengthening is possible, and Void when it is not. Here un $\triangleright$ simply peels off the injection of a defined atom into a VarType.

The crucial property of irrel is that Check (irrel (un $\triangleright D$ ) $\left(D^{+}\right.$D) ) computes to Void. This forbids strengthening a variable of type D out of a term of type D. This is necessary because we cannot satisfy the usual compatibility condition for $\left(D^{+} D\right)$, which would require mapping all variables - including the variable-to-bestrengthened $i$ - to a term of type $D$ that does not mention $i$.

More generally, Check (irrel (un $\triangleright$ D) A) means that variables of type D can never be used to construct terms of type A, which ensures that strengthening never runs into variables of the type being strengthened. The function irrel D A is defined by traversing the graph structure of types (i.e., it unrolls the definitions of defined atoms) and checks not (DefinedAtoms.eq D Dat) for each defined atom Dat it finds.

To account for contravariance, we must define strengthening simultaneously with weakening by irrelevant assumptions, which is similar. About 250 lines of Agda code shows that these two relations together are compatible. Their traversals are then defined by instantiating map twice, mutually recursively — each is passed to the other as map' for the contravariant recursive calls.

\subsection{Weakening}

In addition to weakening by irrelevant types (e.g. weakening a nat with an exp), we can weaken by types that do not appear to the left of a computational arrow (e.g., weakening an exp with an exp).

For a simple version of weakening, the variable relation is similar to strengthening, but uses a different computed condition, and flips the role of $\Psi$ and $\Psi^{\prime}$ (now $\Psi^{\prime}$ is bigger):

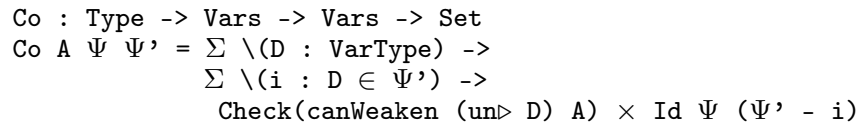

The function canWeaken is a different graph traversal than before: this time, we check irrel (un $\triangleright$ D) A for the left-hand side of each computational arrow A $\supset$ B. Weakening can then be defined using strengthening in contravariant positions, as irrel is exactly the condition that strengthening requires.

This suffices for a simple version of weakening. However, we can be more clever, and observe that types of the form $\forall \Rightarrow$ A are $a l$ ways weakenable, because their proofs are explicitly parametrized over arbitrary extensions of the context. Similarly, $\forall \leq \mathrm{C} \mathrm{A}$ is weakenable with any context composed entirely of C's. Capitalizing on this observation requires a slight generalization of the traversal described above: computationally, weakening $\forall \Rightarrow$ A does not recursively traverse the proof of A, like map usually does, but stops the traversal and instantiates the context quantifier appropriately. Thus, our actual implementation of map is parametrized so that, for each type A, either it is given sufficient information to transform A directly (a function $\langle\Psi>\mathrm{A}->\langle\Psi,>\mathrm{A}$ ), or it has enough information to continue recursively, as in the compatibility conditions described above. We use the former only for weakening the quantifiers (map $<\Psi-i>(\forall \Rightarrow$ A) to $\langle\Psi>(\forall \Rightarrow A)$ ). We refer the reader to our Agda code for details. All told, weakening takes about 210 lines of Agda code to define and prove compatible.

\subsection{Substitution}

Substitution is similar to weakening and strengthening. Its invariant has the same form, using a condition canSubst (un $\triangleright$ D) A. This condition ensures two things: (1) that $D$ is irrelevant to the lefthand-sides of any computational arrow, so that substitution can be defined using weakening-with-irrelevant-assumptions in the contravariant position, and (2) that D is weakenable with all variable types bound by A, so that the term being plugged in for the variable can be weakened as substitution goes under binders. Substitution takes about 220 lines to define and prove compatible.

\section{Related Work}

We have provided comparisons with several other systems throughout the paper: Relative to LF-based systems such as Twelf (Pfenning and Schürmann, 1999), Delphin (Poswolsky and Schürmann, 2008), and Beluga (Pientka, 2008), our framework permits definitions that mix binding and computation; this is essential for defining the datatype sem in the NBE example. Relative to FreshML (Pottier, 2007; Shinwell et al., 2003), our framework enforces invariants about variable scoping in the type system. Such invariants can be proved in Pure FreshML (Pottier, 2007), but we would like to enforce these invariants within a type system, not using an external specification logic.

Aydemir et al. (2008) provide a nice overview of various techniques that are used to implement variable binding, including named, de Bruijn, \{locally / globally\} \{named /nameless $\}$, and weak higher-order abstract syntax (Bucalo et al., 2006; Despeyroux et al., 1995). More recently, Chlipala (2008) has advocated the use of parametric higher-order abstract syntax. We have chosen well-scoped de Bruijn indices (Altenkirch and Reus, 1999; Bellegarde and Hook, 1994; Bird and Paterson, 1999) for our Agda implementation, a simple representation that makes the pronoun structure of variables explicit. It would be interesting to investigate whether any benefits can be obtained by implementing our universe with a different representation. Relative to these techniques for representing binding, the advantage of our framework is that it provides datatype-generic implementations of the structural properties, including substitution. Both the Hybrid frameworks (Ambler et al., 2002; Capretta and Felty, 2007; Momigliano et al., 2007), Hickey et al. (2006)'s work, and Lambda Tamer (Chlipala, 2007) describe languages or tools for specifying data with binding, providing generic implementations of the structural properties. However, to the best of our knowledge, these logical frameworks do not make the computational functions of the meta-language available for use in the framework (except inasmuch as they are, in some cases, used to represent binding itself). In contrast, our universe includes both $\Rightarrow$ and $\supset$.

In this work, we have created a universe of contextual types in Agda. Contextual types appear in Miller and Tiu's work (Miller and Tiu, 2003), as well as in contextual modal type theory (Nanevski et al., 2007). Miller and Tiu's self-dual $\nabla$ connective is closely related to $\Rightarrow$, also capturing the notion of a scoped constant. However, the $\nabla$ proof theory adopts a logic-programming-based distinction between propositions and types, and $\nabla$ binds a scoped term constant in a proposition. In our setting, $\Rightarrow$ allows the meaning of certain propositions (defined atoms) to vary.

Fiore et al. (1999) and Hofmann (1999) give semantic accounts of variable binding. In a sense, the present paper gives a semantics for our type theory, where binding is represented by an indexed inductive definition. However, this semantics does not shed any new light on the datatype-generic definition of the structural properties; it would be interesting to explore a semantic characterization of the conditions under which weakening and substitution are definable.

\section{Conclusion}

In this paper, we have constructed a logical framework supporting datatypes that mix binding and computation: Our framework is implemented as a universe in the dependently typed programming language Agda. Binding is represented in a pronominal manner, so the type system can be used to reason about the scoping of variables. Our implementation provides datatype-generic implementations of 
the structural properties (weakening, subordination-based strengthening, exchange, contraction, and substitution). We have used the framework to program a number of examples, including a scopecorrect version of the normalization-by-evaluation challenge problem discussed by Shinwell et al. (2003). We believe that these examples demonstrate the viability of our approach for simply-typed programming.

We hope also to have clarified the gap between LF-based systems for programming with binding, such as Twelf, Delphin, and Beluga, and a generic dependently typed programming language like Agda. For simply-typed programming, the benefits of the LFbased systems that we were unable to mimic include: (1) the ability to write pronominal variables with a named syntax; and (2) a convenient syntax for applying the structural properties. For example, the syntax of weakening and strengthening is relatively heavy in our setting. In Twelf, weakening is silent, and strengthening (including strengthen? used in the $\eta$-contraction example) is marked by saying which variables do occur, using a non-linear higher-order pattern. In our Agda implementation, weakening must be marked explicitly, and strengthening requires one to enumerate those variables that do not occur instead. However, the more convenient syntax seems within reach for a standalone implementation of our framework; e.g., weakening could be implemented using a form of coercive subtyping.

Of course, one way in which all of the LF-based systems outpace ours is that they support dependent types, which are crucial for representing logics and for mechanizing metatheory. Our most pressing areas of future work are to investigate a dependently typed extension of our universe, and to address the termination issues that we have deferred here. One key issue for the dependently typed version will be the equational behavior of the structural properties, which we have not yet investigated. We would hope that they have the right behavior up to propositional equality (otherwise there is a bug in the code presented here), but it remains to be seen whether we can get Agda's definitional equality to mimic the equations proved automatically by, e.g., Twelf. That said, the fact that the map function defined in Section 4 commutes with all term constructors definitionally in Agda gives us some hope in this regard.

\section{A. Agda Overview}

In this section, we review Agda's syntax, we show a simple example of well-scoped de Bruijn indices, and we give a simple example of a universe. We refer the reader to the Agda Wiki (http://wiki.portal.chalmers.se/agda/) for more introductory materials.

\section{A.1 Well-scoped de Bruijn indices in Agda}

We review the representation of well-scoped de Bruijn indices as an indexed inductive definition (Altenkirch and Reus, 1999; Bellegarde and Hook, 1994; Bird and Paterson, 1999). Agda data types are introduced as follows:

$$
\begin{aligned}
& \text { data List (A: Set) : Set where } \\
& \text { [] : List A } \\
& -:: \text { _ A -> List A } \rightarrow \text { List A }
\end{aligned}
$$

Set classifies Agda classifiers, like the kind type in ML or Haskell. Mixfix constructors are declared by using _ in an identifier; e.g., : : can now be used infix as in Zero : : (Zero : : []).

Functions are defined by pattern-matching:

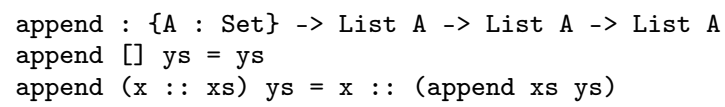

The curly-braces mark an implicit dependent function space. Applications to implicit arguments are not marked in the program; e.g., we do not explicitly apply append to the Set argument A. Agda attempts to infer implicit function arguments and reports an error if they cannot be reconstructed.

Indexed datatypes are defined using a notation similar to GADTs in GHC. For example, we define a datatype $\in$ representing indices into a list:

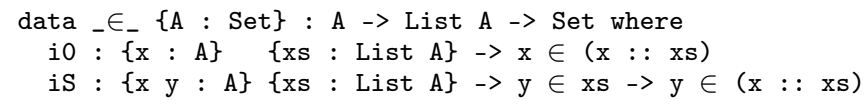

For any Set $A$, and terms $x$ and $x$ s of type $A$ and List $A$, there is a type $x \in \mathrm{xs}$. The first constructor, i0, creates a proof of $\mathrm{x}$ $\in(\mathrm{x}:: \mathrm{xs})$-i.e. $\mathrm{x}$ is the first element of the list. The second constructor iS, creates a proof of $x \in(y:: x s)$ from a proof that $\mathrm{x}$ is in the tail.

As a simple example of dependent pattern matching, we define an $n$-ary version of iS:

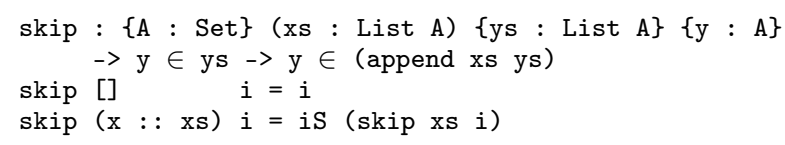

We use an implicit-quantifier for all arguments but the list xs; explicit-quantifiers are written with parentheses instead of curlybraces. The fact that this code type-checks depends on the computational behavior of append; e.g., in the first case, the expression append [] ys reduces to ys, so we can return the index $i$ unchanged.

Well-scoped syntax for the untyped $\lambda$-calculus is defined as follows:

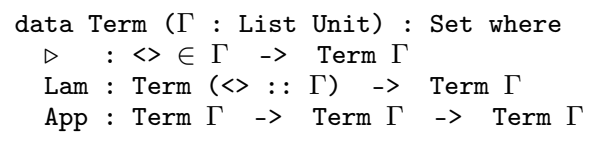

The type Unit is defined to be the record type with no fields, with inhabitant written $\langle>$. We represent variables as indices into a list $\Gamma$ containing elements of the one-element type Unit. (Such lists are isomorphic to natural numbers, but this illustrates the pattern for variables of more than one type.) The constructor $\triangleright$ makes a term from an index into $\Gamma$, which represents a variable. The body of Lam can refer to all of the variables in $\Gamma$, as well as a new bound variable represented by extending $\Gamma$ to $(<>:: \Gamma)$. The $\mathrm{K}$ combinator $\lambda \mathrm{x} . \lambda \mathrm{y} . \mathrm{x}$ is represented as follows: Lam (Lam ( $\triangleright$ (iS i0))). The values of Term $\Gamma$ correspond exactly to the $\lambda$-terms with free variables in $\Gamma$.

\section{A.2 Universes}

A universe is specified by a inductive datatype of codes for types, along with a function mapping each code to a Set. For example, a simple universe with an empty type, a unit type, and binary products is specified as follows:

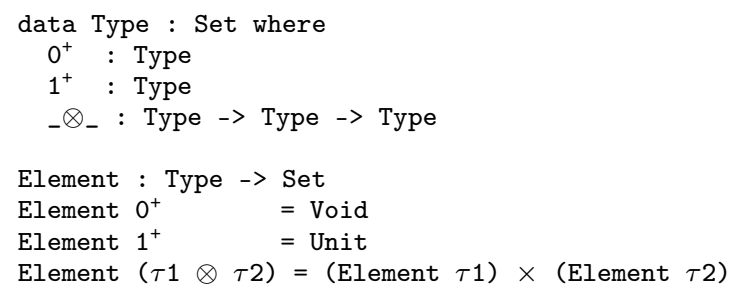

In the right-hand side of Element, we write $A \times B$ for the Agda pair type, etc.

Datatype-generic programs are implemented by recursion over the codes; e.g, every element of the universe can be converted to a string: 


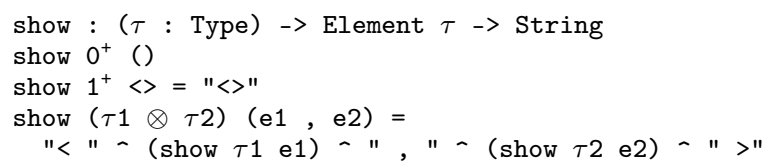

In the first clause, the empty parentheses are a refutation pattern, telling Agda to check that the type in question (in this case Element $\mathrm{O}^{+}$) is uninhabited, and allowing the programmer to elide the right-hand side.

As another example, we will often view booleans as a twoelement universe, with only True inhabited:

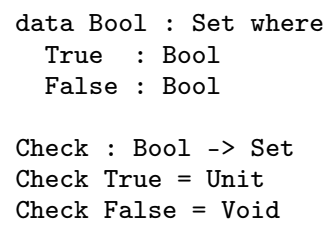

Because Agda implements extensionality for Unit (there is only one record with no fields), terms of type Check True can be left implicit and inferred.

\section{Acknowledgements}

We thank Noam Zeilberger for discussions about this work, and we thank the anonymous reviewers for their helpful feedback on an earlier version of this article.

\section{References}

M. Abbott, T. Altenkirch, and N. Ghani. Containers: constructing strictly positive types. Theoretic Computer Science, 342(1):3-27, 2005.

T. Altenkirch and C. McBride. Generic programming within dependently typed programming. In IFIP TC2 Working Conference on Generic Programming, Schloss Dagstuhl, 2003.

T. Altenkirch and B. Reus. Monadic presentations of lambda terms using generalized inductive types. In CSL 1999: Computer Science Logic. LNCS, Springer-Verlag, 1999.

S. Ambler, R. L. Crole, and A. Momigliano. Combining higher order abstract syntax with tactical theorem proving and (co)induction. In International Conference on Theorem Proving in Higher-Order Logics, pages 13-30, London, UK, 2002. Springer-Verlag.

B. Aydemir, A. Charguéraud, B. C. Pierce, R. Pollack, and S. Weirich. Engineering formal metatheory. In ACM SIGPLAN-SIGACT Symposium on Principles of Programming Languages, pages 3-15, 2008.

F. Bellegarde and J. Hook. Substitution: A formal methods case study using monads and transformations. Science of Computer Programming, 23(23):287-311, 1994.

U. Berger and $\mathrm{H}$. Schwichtenberg. An inverse of the evaluation functional for typed $\lambda$-calculus. In IEEE Symposium on Logic in Computer Science, 1991.

R. S. Bird and R. Paterson. De Bruijn notation as a nested datatype. Journal of Functional Programming, 9(1):77-91, 1999.

A. Bucalo, M. Hofmann, F. Honsell, M. Miculan, and I. Scagnetto. Consistency of the theory of contexts. Journal of Functional Programming, 16 (3):327-395, May 2006.

V. Capretta and A. Felty. Combining de Bruijn indices and higher-order abstract syntax in Coq. In Proceedings of TYPES 2006, volume 4502 of Lecture Notes in Computer Science, pages 63-77. Springer-Verlag, 2007.
A. Chlipala. A certified type-preserving compiler from $\lambda$-calculus to assembly language. In ACM SIGPLAN Conference on Programming Language Design and Implementation, 2007.

A. Chlipala. Parametric higher-order abstract syntax for mechanized semantics. In ACM SIGPLAN International Conference on Functional Programming. ACM, 2008.

K. Crary. Explicit contexts in LF. In International Workshop on Logical Frameworks and Meta-Languages: Theory and Practice, 2008.

J. Despeyroux, A. Felty, and A. Hirschowitz. Higher-order abstract syntax in Coq. In M. Dezani-Ciancaglini and G. Plotkin, editors, International Conference on Typed Lambda Calculi and Applications, volume 902 of Lecture Notes in Computer Science, pages 124-138, Edinburgh, Scotland, 1995. Springer-Verlag.

M. Fiore, G. Plotkin, and D. Turi. Abstract syntax and variable binding. In IEEE Symposium on Logic in Computer Science, 1999.

J. Hickey, A. Nogin, X. Yu, and A. Kopylov. Mechanized meta-reasoning using a hybrid HOAS/de Bruijn representation and reflection. In ACM SIGPLAN International Conference on Functional Programming, pages 172-183, New York, NY, USA, 2006. ACM.

M. Hofmann. Semantical analysis of higher-order abstract syntax. In IEEE Symposium on Logic in Computer Science, 1999.

D. R. Licata, N. Zeilberger, and R. Harper. Focusing on binding and computation. In IEEE Symposium on Logic in Computer Science, 2008.

P. Martin-Löf. An intuitionistic theory of types: Predicative part. In H. Rose and J. Shepherdson, editors, Logic Colloquium. Elsevier, 1975.

C. McBride and J. McKinna. The view from the left. Journal of Functional Programming, 15(1), 2004.

D. Miller and A. F. Tiu. A proof theory for generic judgments: An extended abstract. In IEEE Symposium on Logic in Computer Science, pages 118 $127,2003$.

A. Momigliano, A. Martin, and A. Felty. Two-level hybrid: A system for reasoning using higher-order abstract syntax. In International Workshop on Logical Frameworks and Meta-Languages: Theory and Practice, 2007

A. Nanevski, F. Pfenning, and B. Pientka. Contextual modal type theory. Transactions on Computational Logic, 2007. To appear.

U. Norell. Towards a practical programming language based on dependent type theory. PhD thesis, Chalmers University of Technology, 2007.

F. Pfenning and C. Schürmann. System description: Twelf - a meta-logical framework for deductive systems. In H. Ganzinger, editor, International Conference on Automated Deduction, pages 202-206, 1999.

B. Pientka. A type-theoretic foundation for programming with higher-order abstract syntax and first-class substitutions. In ACM SIGPLAN-SIGACT Symposium on Principles of Programming Languages, pages 371-382, 2008

A. M. Pitts and M. J. Gabbay. A metalanguage for programming with bound names modulo renaming. In R. Backhouse and J. N. Oliveira, editors, Mathematics of Program Construction, volume 1837 of Lecture Notes in Computer Science, pages 230-255. Springer-Verlag, Heidelberg, 2000.

A. Poswolsky and C. Schürmann. Practical programming with higherorder encodings and dependent types. In European Symposium on Programming, 2008.

F. Pottier. Static name control for FreshML. In IEEE Symposium on Logic in Computer Science, 2007.

M. R. Shinwell, A. M. Pitts, and M. J. Gabbay. FreshML: Programming with binders made simple. In ACM SIGPLAN International Conference on Functional Programming, pages 263-274, August 2003.

R. Virga. Higher-Order Rewriting with Dependent Types. $\mathrm{PhD}$ thesis, Carnegie Mellon University, 1999. 